\title{
Resolution of Syncope With Treatment of Sleep Apnea
}

\author{
Floyd B. Willis, MD, Amber L. Isley, MD, Yonas E. Geda, MD, MSc, Ali Shaygan, MD, \\ Luther Quarles, IV, and Paul A. Fredrickson, MD
}

Sleep apnea is a common disorder associated with obesity and related health problems. Although treatment of sleep apnea may relieve some autonomic symptoms, it is currently unknown whether treatment of sleep apnea is specifically associated with the resolution of orthostatism and syncope. Herein we describe a 73-year-old man who had recurrent episodes of syncope. An extensive work-up, including cardiac and neurologic consultations, failed to identify the cause. An objective sleep evaluation led to the diagnosis of sleep apnea. Accordingly, the patient was treated with continuous positive airway pressure, which resolved the syncopal episodes. This case report generates a potentially important hypothesis that recurrent syncope may be effectively treated, in part, by correcting apnea. In patients with recurrent syncope of unknown etiology, a diagnosis of sleep apnea should be considered. ( $\mathrm{J}$ Am Board Fam Med 2008;21:466-468.)

Sleep apnea refers to disrupted ventilation during sleep and is classified as either obstructive or central. ${ }^{1}$ Obstructive sleep apnea (OSA) is the most common sleep breathing disorder, discovered more than 4 decades ago and affecting approximately $20 \%$ of the population. Prevalence estimates of symptomatic moderate-to-severe OSA range from $0.5 \%$ to $4 \%$ in the general population. ${ }^{2,3}$ Symptoms may include loud snoring, witnessed apneas, unrefreshing sleep, daytime somnolence, or impaired cognition. OSA occurs when the upper airways become obstructed because of anatomic factors such as bony abnormalities, soft tissue hypertrophy, and loss of neural reflexes that maintain airway patency during sleep. ${ }^{2,4,5}$

This article was externally peer reviewed.

Submitted 12 December 2007; revised 8 April 2008; accepted 11 April 2008.

From the Department of Family Medicine (FBW, ALI), the Department of Psychiatry and Psychology (PAF), the Department of Internal Medicine (PAF), and the Department of Neurology (LQ), Mayo Clinic, Jacksonville, Florida and the Division of Tertiary Psychiatry and Psychology (YEG), Mayo Clinic, Rochester, Minnesota.

Funding: Dr. Geda was supported in part by K01 MH68351 from the National Institutes of Health.

Conflict of interest: none declared.

Corresponding author: Amber L. Isley, MD, Department of Family Medicine, Mayo Clinic, 4500 San Pablo Road, Jacksonville, FL 32224 (E-mail: isley.amber@mayo.edu.).
Autonomic dysfunction is also associated with OSA and may be manifested by decreased heart rate variability, lower baroreflex sensitivity, increased baseline heart rate, and orthostatic hypotension. ${ }^{6,7}$ Patients with OSA often demonstrate various cardiac dysrhythmias on polysomnography. ${ }^{8}$ Forty percent of syncope is classified as unexplained. ${ }^{9}$ However, it is unknown if true syncope is associated with sleep apnea. In addition, resolution of recurrent syncope with treatment of sleep apnea has not been reported.

\section{Case Report}

A 73-year-old man with a history of hypertension, esophageal reflux, and a body mass index of 35 complained of episodes of weakness on exertion and at rest. His symptoms gradually worsened during a 10 year period and occurred more frequently when he was exposed to heat and with minimal activity, such as light yard work. Unexplained syncopal episodes progressed to monthly events with concomitant emergency department and primary care physician office evaluations. In addition, the patient reported numerous occasions of collapse and syncope for which he did not seek medical attention. Neurology consultation revealed a possible diagnosis of transient ischemic attack and emergency department physicians diagnosed syncope of unknown etiology. Throughout 
his course, he noted gradual memory loss. This prompted neuropsychological memory testing, which was normal.

One syncopal event was witnessed by his family physician. The patient was observed to become diaphoretic without pain or dyspnea and then lost consciousness for 2 minutes. No seizure activity was noted. Glucose monitoring and rhythm strips were normal. The patient fully recovered within 3 minutes of regaining consciousness.

Cardiac evaluation included an event recorder, which documented normal sinus rhythm during a presyncopal event. Holter monitor, electrocardiography, stress testing on a treadmill, polysomnography, and ambulatory blood pressure monitoring showed no evidence of conduction disorder, ischemia, or orthostatic fluctuations in blood pressure associated with apneic events. During hospitalization, telemetry and nursing neurology checks revealed nothing. Neurologic evaluations, including magnetic resonance and computed tomographic imaging of the head, were negative. Electroencephalograms during sleep and while awake showed nonspecific patterns, but during the study, the patient fell asleep while seated and snoring was noted. Further questioning of the patient's spouse revealed that the patient had excessive daytime sleepiness.

The patient underwent overnight oximetry and was found to have 292 desaturation events with a minimum oxygen saturation of $73 \%$ in a pattern consistent with severe OSA. Polysomnography demonstrated loud snoring and an apnea-hypopnea index (the number of apneic and hypopneic episodes divided by the number of hours of sleep) of 73, with no notable effect of sleeping position on apnea-hypopnea index. Treatment with continuous positive airway pressure (CPAP) nasally abolished snoring and the majority of disordered breathing events.

The patient's nasal CPAP compliance was selfreported at $98 \%$. Although objective confirmation was not available, the patient's wife confirmed his compliance report. Daytime alertness improved dramatically with treatment, and repeat overnight oximetry with treatment was normal. Syncope and near-syncopal events completely resolved without recurrence for 1.5 years since the institution of nasal CPAP therapy.

\section{Discussion}

Here we report complete resolution of recurrent syncopal episodes after the diagnosis and treatment of OSA. Before the patient's sleep study he had an extensive work-up, including neurologic and cardiac investigations, that did not identify any known cause for the recurrent syncopal episodes. To our knowledge, this may be the first case that showed complete resolution of recurrent syncope with nasal CPAP treatment. Investigators in Japan reported a patient with OSA who had a marked decrease in the severity of syncope after the institution of nasal CPAP, but they did not observe complete resolution of symptoms. ${ }^{10}$ This case report generates a potentially important hypothesis that recurrent syncope may be effectively treated, in part, by correcting apnea. In patients with recurrent syncope of unknown etiology, a diagnosis of sleep apnea should be considered. We suggest further study using a larger sample to examine the association between recurrent syncopal episodes of unknown etiology and OSA.

\section{References}

1. White DP. Pathogenesis of obstructive and central sleep apnea. Am J Respir Crit Care Med 2005;172: 1363-70. Epub 2005 Aug 11.

2. Pack AI. Advances in sleep-disordered breathing. Am J Respir Crit Care Med 2006;173:7-15. Epub 2005 Nov 10.

3. Young T, Peppard PE, Gottlieb DJ. Epidemiology of obstructive sleep apnea: a population health perspective. Am J Respir Crit Care Med 2002;165: 1217-39.

4. Caples SM, Gami AS, Somers VK. Obstructive sleep apnea. Ann Intern Med 2005;142:187-97.

5. Ryan CM, Bradley TD. Pathogenesis of obstructive sleep apnea. J Appl Physiol 2005;99:2440-50.

6. Ito R, Hamada H, Yokoyama A, et al. Successful treatment of obstructive sleep apnea syndrome improves autonomic nervous system dysfunction. Clin Exp Hypertens 2005;27:259-67.

7. Zakrzewska-Pniewska B, Nojszewska M, Przybylowski T, Byskiniewicz K. Clinical versus electrophysiological assessment of dysautonomia in obstructive sleep apnea syndrome. J Clin Neurophysiol 2004;21:435-9.

8. Caples SM, Rosen CL, Shen WK, et al. The scoring of cardiac events during sleep. J Clin Sleep Med 2007;3:147-54.

9. Strickberger SA, Benson DW, Biaggioni I, et al. AHA/ACCF scientific statement on the evaluation of syncope: from the American Heart Association Councils on Clinical Cardiology, Cardiovascular 
Nursing, Cardiovascular Disease in the Young, and Stroke, and the Quality of Care and Outcomes Research Interdisciplinary Working Group; and the American College of Cardiology Foundation in Collaboration with the Heart Rhythm Society. J Am Coll Cardiol 2006;47:473-84.
10. Hamada E, Okamoto K, Okuda B. A case of pure autonomic failure (PAF) with sleep apnea syndrome (SAS) and successful treatment of dysautonomia with nasal continuous positive airway pressure (CPAP). [Article in Japanese]. Nippon Ronen Igakkai Zasshi 2004:41:233-6. 